\title{
IT-Management und IT-Strategie
}

\author{
Susanne Strahringer · Thomas Widjaja
}

(C) Springer Fachmedien Wiesbaden 2017

Das IT-Management und die IT-Strategieentwicklung im Rahmen der digitalen Transformation konfrontieren Unternehmen mit zahlreichen neuen Fragestellungen. Die neun praxisorientierten Beiträge dieses Schwerpunkthefts veranschaulichen diese Entwicklung, zeigen die damit verbundenen organisatorischen und ökonomischen Herausforderungen auf und bieten konkrete Hinweise zu deren Bewältigung. Hierbei werden drei übergeordnete Themenfelder adressiert: Zunächst werden die Veränderungen der organisatorischen Einbettung des IT-Managements im Rahmen der digitalen Transformation beleuchtet. Zweitens, wird aufgezeigt, wie und mit welchen Methoden IT möglichst wertstiftend gesteuert werden kann. Drittens, diskutieren zwei Beiträge neue Herausforderungen für das IT-Management und die IT-Strategieentwicklung, die sich aus der Nutzung von Cloud-Computing ergeben.

Die Auswirkung der digitalen Transformation auf die Rolle des IT-Managements in Organisationen - das erste der drei Themenfelder - wird in den Beiträgen eins bis drei unseres Schwerpunkthefts behandelt. Der erste Artikel zeichnet ein Bild der „IT-Organisation der Zukunft“ und schlägt IT-Führungskräften sieben konkrete Handlungsfelder vor. Der nächste Beitrag fokussiert auf das Management des Prozesses der digitalen Transformation und leitet auf Basis einer Literaturrecherche, einer Umfrage unter 110 Unternehmen und drei Fallstudien eine Liste von Managementaufgaben für die Gestaltung dieses Transformationsprozesses ab. Der dritte Artikel unseres Hefts beschäftigt sich mit der aufkommenden Rolle des Chief Digital Officers - auf Grundlage einer umfassenden Analyse von Stellenanzeigen

\footnotetext{
S. Strahringer

Technische Universität Dresden, Dresden, Deutschland

E-Mail: susanne.strahringer@tu-dresden.de

T. Widjaja $(\triangle)$

Universität Passau, Passau, Deutschland

E-Mail: thomas.widjaja@uni-passau.de
} 
sowie Experteninterviews werden die Aufgabenbereiche eines typischen Chief Digital Officers und die damit verbundenen Anforderungen identifiziert. In Summe verdeutlichen diese drei Beiträge die Vielfältigkeit der neuen Herausforderungen bei der Suche nach einer angemessenen organisatorischen Einbettung von IT-Management und IT-Strategieentwicklung.

Die Beiträge vier bis sieben beschäftigen sich mit der Frage, wie IT-Management und IT-Strategieentwicklung dazu beitragen können, die Unternehmens-IT und ITProjekte möglichst wertstiftend auszurichten. Der vierte Beitrag unterstützt Führungskräfte bei der ökonomischen Bewertung von Digitalisierungsprojekten. Diese Frage ist vor dem Hintergrund der Vielzahl von aktuell durchgeführten Digitalisierungsprojekten und deren oftmals unklarem konkreten Wertbeitrag von hoher Praxisrelevanz. Das Kernergebnis des Beitrags ist ein Bewertungsschema und ein daraus abgeleitetes Vorgehensmodell zur Identifikation des optimalen Digitalisierungsportfolios. Der nächste Beitrag stellt mit „CIOverview“ ein durch die swissICT Fachgruppe IT-Service-Management entwickeltes Rahmenwerk zur wertschöpfungsorientierten Steuerung der IT vor. Unser sechster Beitrag beschäftigt sich mit der Frage, wie das IT-Controlling auf die enge Verbindung der Fachbereiche mit der ITOrganisation im Rahmen der digitalen Transformation reagieren kann und zeigt entsprechenden Handlungsbedarf auf. Im siebten Beitrag wird kritisch hinterfragt wie objektiv die Erfolgswahrnehmung von IT-Projekten tatsächlich ist. Insgesamt zeigt sich in diesen Beiträgen, dass auch bisweilen gut etablierte IT-Management-Methoden und Verfahren zur IT-Strategieentwicklung nicht unverändert bleiben können, sondern regelmäßig zu verfeinern und anzupassen sind.

Die Beiträge acht und neun behandeln das Thema Cloud-Computing und die damit verbundenen Herausforderungen für das IT-Management. Im achten Beitrag wird das Konzept der Rebound-Effekte auf den Cloud-Computing-Kontext übertragen. Rebound-Effekte beschreiben Situationen, in denen eine Technologie zwar grundsätzlich Einsparpotenzial ermöglicht, aber aufgrund von beispielsweise erhöhter Nutzungsintensität die Einsparungen nicht realisiert werden können. Die Kenntnis solcher Effekte hat interessante Implikationen für das IT-Management im Kontext von Cloud-Computing. Der neunte Beitrag nimmt die Perspektive von CloudPlattformanbietern ein und behandelt die Frage, wie sich diese Cloud-Plattformanbieter die Loyalität der unabhängigen Softwareanbieter sichern können. Beide Beiträge verdeutlichen, welche neuen Fragestellungen an das IT-Management aus dem Einsatz von Cloud-Computing resultieren und zeigen entsprechende Lösungsansätze auf.

Bei der Gewinnung von Beiträgen für unser Schwerpunktheft wurde neben dem üblichen Verfahren erstmals ein neuer Weg beschritten, indem gezielt Beiträge aus einer Tagung für die HMD ausgewählt wurden. Hierfür wurde der von uns begleitete, namensgleiche Track „IT-Management und IT-Strategie“ auf der diesjährigen Internationalen Tagung Wirtschaftsinformatik in St. Gallen genutzt. Aus der Menge der in diesem Track präsentierten Beiträge wurden besonders geeignete identifiziert und anschließend von den Autorinnen und Autoren im Rahmen eines so genannten Fast-Track-Verfahrens für die HMD zielgruppengerecht überarbeitet. Auf diesem Wege sind die Beiträge sieben, acht und neun für unser Schwerpunktheft gewonnen worden. 
Alle Beiträge des Heftes behandeln die Themen IT-Management und IT-Strategie aus Sicht von Wissenschaft und Praxis - die Autorinnen und Autoren decken zwar zahlreiche aktuelle Herausforderungen auf, geben aber auch konkrete Handlungsempfehlungen. Ihnen, liebe Leserinnen und Leser, wünschen wir im Namen aller, die zu diesem Heft beigetragen haben, viel Freude und Inspiration bei der Lektüre! 